\title{
An Unusual Case of Dyspnea
}

\section{Philip David Houck ${ }^{*}$}

Baylor Scott \& White Healthcare, USA

*Corresponding author: Philip David Houck, Staff Cardiologist, Baylor Scott \& White Healthcare, Cardiology 2401 S 31 st street Temple, Texas 76508, USA, Tel: 1512 966 4475; Fax: 1254724 2661; E-mail: phouck@sw.org

Rec date: Jul 6, 2014; Acc date: Aug 12, 2014; Pub date: Aug 26, 2014

Copyright: (C) 2014 Philip David Houck. This is an open-access article distributed under the terms of the Creative Commons Attribution License, which permits unrestricted use, distribution, and reproduction in any medium, provided the original author and source are credited.

\begin{abstract}
Dyspnea is the perception that breathing is not easy. Generally the heart or lung is blamed for this malady. There are other causes. Every organ of the body can cause shortness of breath. The case presented exemplifies the search for dyspnea involves investigation of multiple organs with multiple maneuvers.
\end{abstract}

Keywords: Dyspnea; Organs; Heart; Lungs; Patients

\section{Introduction to Dyspnea}

\section{Heart and lung}

Dyspnea is the perception that breathing is not easy. Generally, the heart or lung is blamed for this malady. Heart failure, either diastolic or systolic, causes dyspnea by increasing the fluid weight of the lungs. The diaphragm has increased work to elevate and lower the heavy water filled lungs. This extra work is perceived as dyspnea [1]. Heart failure patients have learned to ease this discomfort by sitting up to lower their preload thereby draining the fluid into the venous system $[2,3]$. During activity ischemic patients increase heart rate and blood pressure and elevate filling pressures due to diastolic dysfunction. These patients slow down their physical efforts to lessen the ischemic burden and relieve the elevated filling pressures.

Lung patients present a different set of problems that also increase the work of the breathing. Reactive airway disease requires longer and more forceful exhalation to overcome the smaller airways [4]. Emphysema and barrel chest deformation change the mechanics of the diaphragm and efficiency of ventilation is compromised. Lung cancer patients have occluded lymphatic vessels or blocked airways that cause ventilation perfusion mismatches. There are also other causes of dyspnea not related to the heart or lungs.

\section{Other organ causes}

The kidney is often neglected in the workup of dyspnea. After expensive cardiac catheterization, lung volumes, high resolution computed tomography the bicarbonate is found to be low due to renal tubular acidosis [5]. Baking soda is a very cheap fix to this cause of dyspnea.

The brain is complex beyond comprehension and via parasympathetic, sympathetic, vagal, direct innervation, and hormonal peptides controls all of the organs.

The brain can alter respiratory status. Anytime a patient has spells that are disturbing with a negative emergency room visit, hyperventilation should be excluded as the cause. This can be simply tested by forced hyperventilation for 1 to 2 minutes to see if the symptoms are reproduced. These symptoms include tingling around lips and fingers with a miserable light headed feeling of pre syncope and a fearful dread aura. Hyperventilation is always safer than cardiac catheterization and should be performed in any patient who has confusing symptoms prior to an invasive procedure.

Gastrointestinal and hematopoietic systems can contribute to dyspnea. The liver can cause a central hyperventilation when it fails and ammonia levels rise. These patients are not complaining of dyspnea since they are usually in a comatose state. Watery stools from diarrhoea cause the loss of both potassium and bicarbonate inducing a hyperchloremic acidosis. This result will give patients shortness of breath in an attempt to correct the acidosis. Anemia will increase the cardiac output and any additional stress will cause dyspnea. All of the organs can contribute to shortness of breath.

\section{External stressors contributing to shortness of breath}

Excess consumption promotes storage into blood vessels adding to hypertension and diastolic stiffness of vessels. Muscular deconditioning is often culprit in our obese patients and contributes to further obesity [6]. Often there is a preceding injury from a year ago that caused inactivity and a 50 pound weight gain. The patient can no longer tolerate previous easily performed activities. The temptation to ask "a year ago if you carried 50 pounds up the stairs would you be short of breath?" is great. For patient relations this question should be avoided. Advice should include an exercise prescription as well as weight loss. The exercise prescription should be directed toward their injury with encouragement and hope that they can improve with hard and sometimes painful muscular conditioning.

Dehydration causes shortness of breath and doctors can be the blame. Patients with peripheral edema from venous insufficiency are prescribed a diuretic. The patient becomes intravascularly dehydrated with continued edema fluid and now has shortness of breath due to low cardiac output and ventilation perfusion mismatch. They revisit their doctor and are prescribed more diuretic. Renal failure and referral to a specialist is in the patient's future. There are many other causes including the obvious of pulmonary embolism with ventilation and perfusion mismatch and chronotropic incompetence. Even obstructive sleep apnea can make people sluggish tired and feel they have increased effort in their activities. Occasionally medications like the new oral anticoagulants can give the perception of shortness of breath. 


\section{The puzzle}

Recently, an 84 year old female was referred to me for dyspnea by a partner after an extensive workup by himself and pulmonary consultants. The patient was referred for a 2 nd opinion relative to significant dyspnea. She did not experience orthopnea or PND, and was basically fine at rest until she attempted any type of activity. Getting up and going to the restroom, or walking several steps would cause significant dyspnea.

Physical examination did not reveal a cause of this degree of dyspnea. Vital signs included a blood pressure of $145 / 85 \mathrm{mmHg}$, a pulse of 68 beats per minute, a saturation of $97 \%$ and she was overweight at 187 pounds. Palpebrae were pink consistent with adequate hemoglobin. Jugular venous distension was estimated below $5 \mathrm{~cm}$ without Kussmaul's inspiratory rise. Carotid upstroke was full suggesting adequate cardiac output. There was a slight pulmonary tap during expiration suggesting elevated pulmonary pressures. The PMI was not displaced and discrete suggesting normal systolic function and probable hypertensive heart disease. Auscultation revealed an S1 to be crisp and S2 was normally split suggesting no evidence for cardiac dyssynchrony and normal systolic function. There was an S4 consistent with diastolic dysfunction and a soft outflow murmur consistent with a sclerotic aortic valve. Lung examination was clear with normal expiratory phase. There was no edema and skin was tented suggesting volume depletion. Summary of the examination was normal systolic function with diastolic dysfunction and over diuresis.

The patient had a previous workup that included lung volumes, computed tomography of the chest, right and left cardiac catheterization, which had not demonstrated a reason for the patient's significant dyspnea. The patient noted she felt very well on her 1st Holter monitor, where her average heart rate was 67 beats per minute (bpm). She had a repeat Holter monitor while feeling poorly, with an average heart rate of $56 \mathrm{bpm}$. Maximum heart rate obtained on Holter monitor was $80 \mathrm{bpm}$ with a minimum of 33 . The patient's echocardiogram demonstrated hypertrophy with normal systolic function and restrictive inflow pattern consistent with diastolic dysfunction. The patient also had a cardiac catheterization where she had right atrial pressures of $15 \mathrm{mmHg}$, right ventricular pressures $15 / 13 \mathrm{mmHg}$ with a mean of $21 \mathrm{mmHg}$, pulmonary artery was $48 / 17$ $\mathrm{mmHg}$ with a mean of $26 \mathrm{mmHg}$, pulmonary catheter wedge pressure was $23 \mathrm{mmHg}$, but fell to $14 \mathrm{mmHg}$ after nitroglycerin. She did not have any substantial coronary artery disease with a previous stent to the right coronary artery that was patent. She had a renal artery stenosis detected with a $70 \%$ right renal stenosis with previous right renal artery stenting. Peripheral vascular disease was present with a $50 \%$ left popliteal stenosis, $100 \%$ tibial occlusion. Laboratory revealed bicarbonate of $21 \mathrm{mEq} / \mathrm{L}$.

The initial impression of this patient's problem appears possibly to be bradycardia related, along with volume depletion, and some degree of renal tubular acidosis with chronic bicarbonate in the low 20s. She certainly had diastolic dysfunction with evidence for a stiff ventricle. The patient's assessment was she had increasing dyspnea with less activity and wanted help with her affliction.

\section{Maneuvers performed}

Furosemide and beta blocker was discontinued. Salt restriction was emphasized. The heart rate increased to an average heart rate of 72 bpm with no improvement in dyspnea. Volume status was euvolemic with a rise in blood pressure to $170 / 80$. Spironolactone was added for hypertension with control of blood pressure and still no improvement in dyspnea. A tablespoon of sodium bicarbonate corrected metabolic acidosis with no improvement in dyspnea. Finally, Ranolazine was added for diastolic dysfunction (off label property of Ranolazine) with failure to improve the patient [7]. Another holter was performed to investigate occult chronotropic incompetence - none was found. The chump was stumped.

\section{Doctoring}

Doctoring should be defined as the ability to make a patient better when there is no known pharmacology or intervention for their affliction. Patients can become frustrated with doctors when doctors fail to find the solution. They are passed from one specialist to another. Patients may feel that their complaint is not taken seriously or that the doctor thinks they are a psychological misfit. Hopelessness can be expressed as anger or despair. Doctors who have no other place to send the patient can have the same anger, despair, and depression. The next maneuver was doctoring; reassuring the patient that they believed in their complaint and had not given up in finding a solution. The plan was to gradually increase exercise and be re-evaluated in one month. A big and confident smile and a humorous statement should send the patient out of the door smiling.

The patient presented before her clinic appointment in extremis with renal failure documented by creatinine greater than $4 \mathrm{mg} / \mathrm{dL}$, with normal potassium. The renal failure occurred over one week. She was in a junctional rhythm, mildly hypotensive. She was admitted for a pacemaker, renal failure and a definitive bedside procedure was performed.

\section{Procedure}

Foley catheter drained $3000 \mathrm{cc}$ of urine. She had three bladder surgeries and suffered from bladder obstruction. The obstruction caused renal tubular acidosis, bradycardia from vagal tone and renal failure. The Foley cured the renal failure, renal tubular acidosis, and vagally mediated bradycardia. It was decided to place a dual chamber pacemaker since the probability of future bladder obstruction was likely. The patient was re-evaluated in the clinic and was improved in her breathing but not compliant with intermittent straight catheterizations. She still had moderate dyspnea and fatigue with activity. The pacemaker was set at $72 \mathrm{bpm}$ as per factory settings. The pacemaker was reprogramed to a heart rate of $85 \mathrm{bpm}$. Restrictive cardiac disease can be thought of as the ventricle being already full. With a full ventricle the mitral inflow occurs over a very short time interval due to elevated diastolic pressures. One therapy for restrictive disease is to empty the ventricle more frequently by raising the heart rate. This will improve cardiac output and lower filling pressures by empting the full ventricle more frequently. At a heart rate of 85 the patient could do all of her activities without limiting dyspnea. Previously, an obstructed bladder increased vagal tone blunting heart rate response. The lower heart rate and restrictive filling caused dyspnea.

\section{References}

1. Clark AL (2006) Origin of symptoms in chronic heart failure. Heart 92: 12-16.

2. Houck PD (2013) Alternative view of congestive heart failure exacerbations: Role of lymphatic function and inflammation. OA Med Hypothesis 1: 6 . 
Citation: Houck PD (2014) An Unusual Case of Dyspnea. Primary Health Care 4: 169. doi:10.4172/2167-1079.1000169

Page 3 of 3

3. Houck PD (2013) Evolution of heart failure: Crotchety old cardiologist. OA Med Hypothesis 1: 7.

4. Boulet LP, Turcotte H (2007) Lung hyperinflation, perception of bronchoconstriction and airway hyperresponsiveness. Clin Invest Med 30: 2-11.

5. Bowman JS, Peckler BF (2002) Type IV renal tubular acidosis presenting as dyspnea in two older patients taking angiotensin-converting enzyme inhibitors. Annuals of emergency Medicine 39: 73-76.
6. Sin DD, Jones RL, Man SF (2002) Obesity is a risk factor for dyspnea but not for airflow obstruction. Arch Intern Med 162: 1477-1481.

7. Lovelock JD, Monasky MM, Jeong EM, Lardin HA, Liu H, et al. (2012) Ranolazine improves cardiac diastolic dysfunction through modulation of myofilament calcium sensitivity. Circ Res 110: 841-850. 